\title{
Molecular typing, and integron and associated gene cassette analyses in Acinetobacter baumannii strains isolated from clinical samples
}

\author{
LINGQING XU ${ }^{1}$, SHUFEI DENG ${ }^{1}$, WEIHONG WEN ${ }^{1}$, YINGXIAN TANG ${ }^{1}$, LINJUAN CHEN ${ }^{1}$, \\ YUZHEN LI ${ }^{1}$, GUOQUAN ZHONG ${ }^{1}$, JIEHUA LI ${ }^{1}$, WEI-JEN TING ${ }^{1}$ and BISHI FU ${ }^{1,2}$ \\ ${ }^{1}$ Department of Clinical Laboratory, The Sixth Affiliated Hospital of Guangzhou Medical \\ University, Qingyuan People's Hospital, Qingyuan, Guangdong 511518; ${ }^{2}$ School of Basic Medical \\ Sciences, Guangzhou Medical University, Guangzhou, Guangdong 511436, P.R. China
}

Received July 11, 2019; Accepted March 18, 2020

DOI: 10.3892/etm.2020.8911

\begin{abstract}
The present study aimed to investigate the association between drug resistance and class I, II and III integrons in Acinetobacter baumannii (ABA). Multilocus sequence typing (MLST) is a tool used to analyze the homology among house-keeping gene clusters in ABA and ABA prevalence and further provides a theoretical basis for hospitals to control ABA infections. A total of 96 clinical isolates of non-repeating ABA were harvested, including 74 carbapenem-resistant ABA (CRABA) and 22 non-CRABA strains, and used for bacterial identification and drug susceptibility analysis. Variable regions were sequenced and analyzed. Then, 7 pairs of housekeeping genes were amplified and sequenced via MLST and sequence alignment was performed against the Pub MLST database to determine sequence types (STs) strains and construct different genotypic evolutionary diagrams. The detection rate of CRABA class I integrons was $13.51 \%$ (10/74); no class II and III integrons were detected. However, class I, II and III integrons were not detected in non-CRABA strains. The variable regions of 9 of 10 class I integrons were amplified and 10 gene cassettes including aacC1, aac1, aadDA1, aadA1a, aacA4, dfrA17, aadA5, aadA1, aadA22 and aadA23 were associated with drug resistance. The 96 ABA strains were divided into 21 STs: 74 CRABA strains containing 9 STs, primarily ST208 and ST1145 and 22 non-CRABA strains containing 18 STs, primarily ST1145. Class I integrons are a critical factor underlying drug resistance in ABA. CRABA and non-CRABA strains differ significantly; the former primarily contained ST208 and ST1145, and the latter contained ST1145. Most STs
\end{abstract}

Correspondence to: Dr Bishi Fu, School of Basic Medical Sciences, Guangzhou Medical University, 1 Guangyi Road, Panyu, Guangzhou, Guangdong 511436, P.R. China

E-mail: fubishi@gzhmu.edu.cn

Key words: Acinetobacter baumannii, integrons, variable region, multilocus sequence typing, drug resistance were concentrated in intensive care units (ICUs) and the department of Neurology, with the patients from the ICUs being the most susceptible to bacterial infection. In the Sixth Affiliated Hospital of Guangzhou Medical University, Qingyuan People's Hospital, ABA is potentially horizontally transmitted and MLST can be used for clinical ABA genotyping.

\section{Introduction}

Acinetobacter baumannii (ABA) is an important conditional pathogen in hospitals, and often causes infection in critically ill patients $(1,2)$. Carbapenems have always been considered as an important class of antibiotics to treat various infections. However, the proportion of carbapenem-resistant A. baumannii (CRABA) has gradually increased in recent years (3). Data from the China Antimicrobial Surveillance Network (2016) indicate that ABA ranked third among all the isolates (4). From 2005 to 2016, the resistance rate of ABA to imipenem rose from $31.0-68.6 \%$ and that to meropenem rose from $39.0-71.4 \%(5)$.

Integrons are closely associated with the multidrug resistance of ABA (1). Integron cassettes are mobile genetic materials in the bacterial genome that carry components of site-specific recombination systems that can integrate a number of drug resistance gene cassettes together to form multiple drug resistance (6). There are currently 10 types of integrons that have been discovered and identified in humans, but only 5 integrons are associated with drug resistance gene cassettes (7). Class I integrons are the commonest type and their structures include 5 ' and 3 ' conserved ends and an internal variable region encoding antibiotic resistance genes (2). The gene sequence of class II integrons is similar to that of class I integrons; the encoded integrin has $46 \%$ homology with class I. It is located on transposon $\operatorname{Tn} 7$ and its derivatives and is a defective DNA integrase Intl gene (6), Class III integrons have been identified on a resistant plasmid of Serratia marcescens that is resistant to imipenem. Its integrase gene encodes 320 amino acids and has $51 \%$ homology with integrase I (8). Class IV integrons are a special type of integrons: They were discovered by Cambray et al (9) from Vibrio cholerae. These 
integrons are $126 \mathrm{~kb}$ in length and contain $\geq 179$ genes. The primary function of these integrons is to encode adaptability genes. Class V integrons are located on a complex transposon carried by Vibrio salmonicidum (10).

At present, studies are mainly focusing on type I, II and III integrons, as they serve a vital role in drug resistance transmission. The product encoded by them can resist most clinical antibiotics (10). Those antibiotics can be divided into nine categories, of which class A are carbocylase dilutes: Imipenem (IPM) and meropenem (MEM); class $B$ are aminoglycosides: Gentamicin (GM) and amikacin, (AN); class $\mathrm{C}$ are quinolones: Ciprofloxacin (CIP), levofloxacin (LVX), and ciprofloxacin (MXF); class D are cephalosporins: Cefazoxime (CZ), ceftazidime (CAZ), cefotaxime (CTX) and cefepime (FEP); class E are broad-spectrum penicillins: Ampicillin/sulbactam (SAM), piperacillin/tazobactam (TZP), via ampicillin (AMC) and piperacillin (PIP); class $F$ are $\beta$-lactams: Aztreonam (ATM) and amoxicillin/ clavulanic acid (AM); class G are sulfonamides: Compound neomin (SXT); class $\mathrm{H}$ are tetracyclinea: Tetracycline (TE); and class I are amides and alcohols: Chloramphenicol (C). The resistance rate of integron-positive strains to $\beta$-lactams, aminoglycosides and quinolone antibiotics is increased compared with integron-negative strains (7). In China, integrons with resistance gene cassettes are considered as the main drug resistance factor, as the detection rate of integrons is between $52.3-69.6 \%$ (11).

The present study collected CRABA and non-CRABA clinical samples from different clinical departments in the Sixth Affiliated Hospital of Guangzhou Medical University, investigated the association between integron distribution and drug resistance in ABA, and analyzed the homology among multilocus sequence types (MLSTs) in ABA. ABA integrase genes, variable regions and 7 housekeeping genes from 96 strains were amplified and sequenced.

\section{Materials and methods}

Strain conservation. A total of 96 ABA strains were harvested and separated from clinical samples between August 2014 and October 2018, and duplicates were eliminated. Samples were obtained from the Intensive Care Unit (ICU), and departments of Neurology, Infectious Diseases, Respiratory Medicine, Hematology, neurosurgery, Urology, Neonatal and ENT. All the experiment are performed under the permission of Medical Ethics committee of Qingyuan People's Hospital. Quality control strains were Escherichia coli ATCC25922, Staphylococcus aureus ATCC25923 and Pseudomonas aeruginosa ATCC27853 (American Type Culture Collection). Among the 96 ABA strains, 74 were CRABA strains and 22 were non-CRABA strains (Fig. 1). Written informed consent was obtained from all patients.

Instruments and reagents. Instruments included a Phoenix M50 automatic bacterial detector (Becton, Dickinson and Company), a T100 Thermal Cycler (Bio-Rad Laboratories, Inc.) and a Gel Dox XR+gel imaging system (Bio-Rad Laboratories, Inc.). Reagents included Colombia blood Agar plate (Guangzhou Dijing Microbiology Co., Ltd), bacterial group DNA extraction kit, 2X Taq PCR MasterMix, Gel Red nucleic acid dye and Marker I, II, III DNA Ladder (all from Tiangen Biotech (Beijing) Co., Ltd.) and primers from Shanghai Shenggong Bioengineering Technology Service Co., Ltd.

Total DNA extraction from bacteria. The strains were stored in sterile milk at $4^{\circ} \mathrm{C}$ and resuscitated prior to use. Following separation and culturing on blood-containing media, a sufficient number of bacterial colonies was selected with aseptic cotton and inoculated in a $1.5-\mathrm{ml}$ sterile saline Eppendorf (EP) tube to prepare a bacterial suspension and DNA was extracted in accordance with the protocol of the DNA extraction kit, and transferred to aseptic $1.5 \mathrm{ml}$ EP tube and stored at $-80^{\circ} \mathrm{C}$.

Primer design. Primers were designed as described previously $(12,13)$ and are listed in Tables I and II.

PCR for integrase genes. The PCR mix for class I, II and III integrons comprised: $12.5 \mu \mathrm{l} 2 \mathrm{X}$ Taq PCR MasterMix, including Taq DNA polymerase, dNTPs, reaction buffer and $\mathrm{MgCl}_{2}, 0.5 \mu \mathrm{l}$ each of the forward and reverse

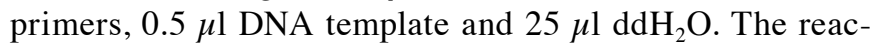
tion conditions were: Initial denaturation at $95^{\circ} \mathrm{C}$ for $5 \mathrm{~min}$, followed by 26 cycles of denaturation at $94^{\circ} \mathrm{C}$ for $30 \mathrm{sec}$, annealing at $62^{\circ} \mathrm{C}$ for $30 \mathrm{sec}$ and extension at $72^{\circ} \mathrm{C}$ for $1 \mathrm{~min}$, and then a final extension at $72^{\circ} \mathrm{C}$ for $5 \mathrm{~min}$. The PCR products were visualized electrophoretically on a $2 \%$ agarose gel for $45 \mathrm{~min}$ at $80 \mathrm{~V}$, and visualized using a gel imaging system (Bio-Rad Laboratories, Inc.).

PCR for the variable region. The PCR mix for variable regions of class I, II and III integrons comprised: $12.5 \mu \mathrm{l} 2 \mathrm{X}$ Taq PCR MasterMix, $0.5 \mu \mathrm{l}$ each of the forward and reverse primer, $1 \mu \mathrm{l}$

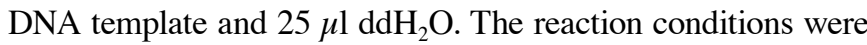
as follows: Initial denaturation at $94^{\circ} \mathrm{C}$ for $5 \mathrm{~min}$, followed by 35 cycles of denaturation at $94^{\circ} \mathrm{C}$ for $30 \mathrm{sec}$, annealing at $62^{\circ} \mathrm{C}$ for $30 \mathrm{sec}$ and extension at $72^{\circ} \mathrm{C}$ for $2 \mathrm{~min}$, and a final extension at $72^{\circ} \mathrm{C}$ for $7 \mathrm{~min}$. The PCR products were visualized electrophoretically on a $1.2 \%$ agarose gel for $35 \mathrm{~min}$ at $80 \mathrm{~V}$ and visualized using a gel imaging system (Bio-Rad Laboratories, Inc.).

PCR for 7 housekeeping genes in MLSTs. The PCR mix for 7 housekeeping genes was: $12.5 \mu 12 \mathrm{X}$ Taq PCR MasterMix, $0.5 \mu \mathrm{l}$ each of the forward and reverse primer, $1 \mu \mathrm{l}$ DNA

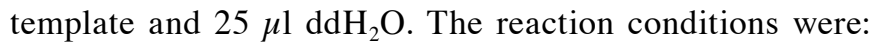
Initial denaturation at $94^{\circ} \mathrm{C}$ for $5 \mathrm{~min} ; 30$ cycles of denaturation at $94^{\circ} \mathrm{C}$ for $1 \mathrm{~min}$, annealing at $56^{\circ} \mathrm{C}$ for $1 \mathrm{~min}$ and extension at $72^{\circ} \mathrm{C}$ for $2 \mathrm{~min}$; final extension at $72^{\circ} \mathrm{C}$ for $7 \mathrm{~min}$. The PCR products were visualized electrophoretically on a $1 \%$ agarose gel (35 min, $65 \mathrm{~V}$ ) and visualized using a gel imaging system (Bio-Rad Laboratories, Inc.).

Sequence analysis of the variable regions and 7 housekeeping genes. The PCR products (cut-off value >2) were sent to Shanghai Bioengineering Company for sequencing, and reads were corrected and spliced using Chromas software (version 2.6.6; http://technelysium. com.au/wp/chromas/) and compared and analyzed using BLAST (BLAST+ version 2.10.0; https://blast.ncbi.nlm. 


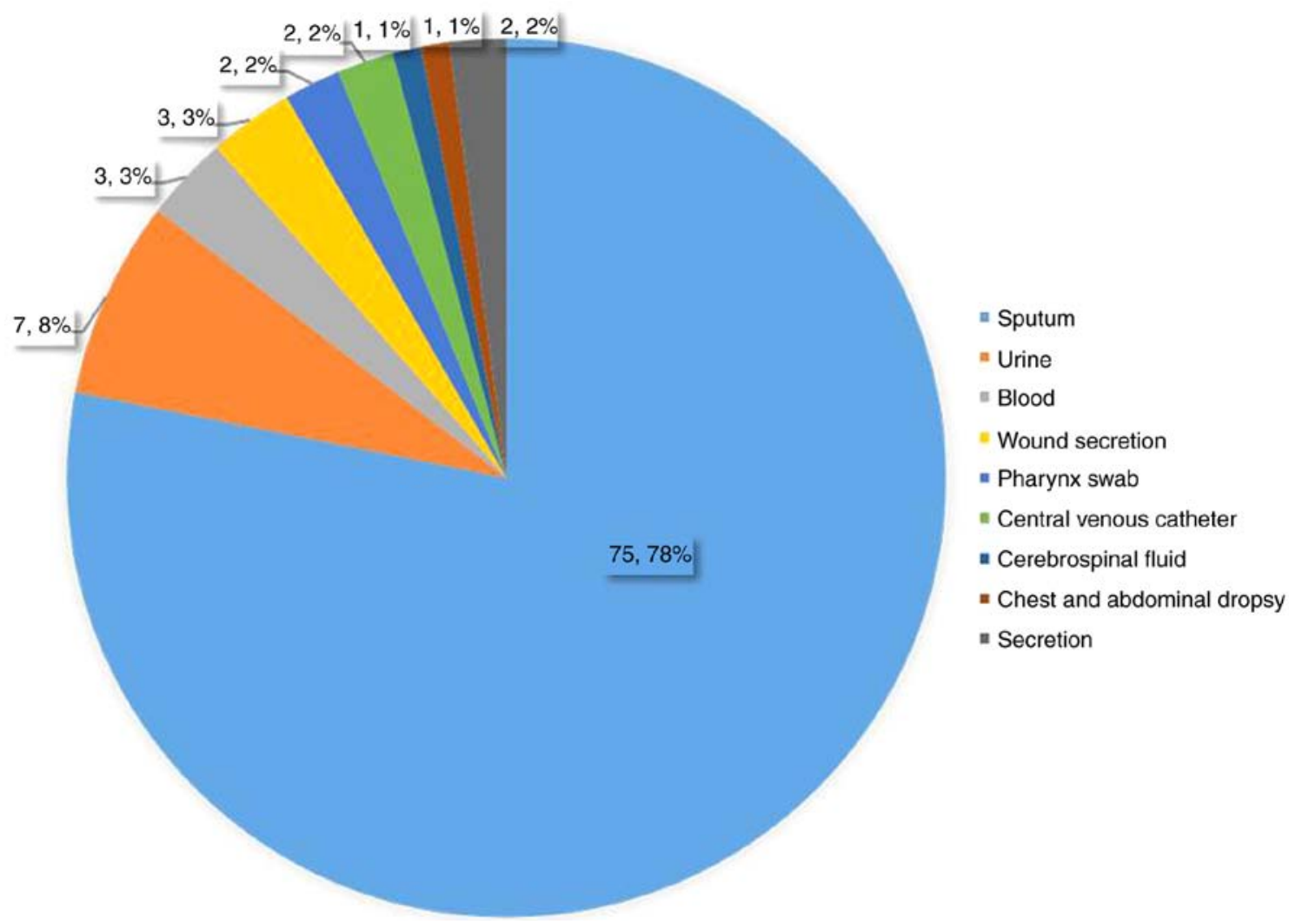

Figure 1. Distribution of 96 Acinetobacter baumannii strains in sample types.

nih.gov/Blast.cgi) and reads with the highest degree of coincidence were selected.

Genotyping analysis of ABA MLSTs. From the ABA MLST database (https://pubmlst.org/abaumannii/), the sequencing reads were subjected to BLAST analysis and different allele values were obtained. All strains were ranked, thus yielding an allelic spectrum in the order of gltA, gyrB, gdhB, recA, cpn60, gpi and rpoD.

Whole-genome sequencing. Genomic DNA was extracted from the culture with a QIAamp DNA kit according to the manufacturer's instructions (Qiagen). Purified DNA was fragmented by sonication with a Covaris S2 instrument (Covaris, Inc.). Indexed shotgun sequencing libraries were prepared using the Kapa Library Preparation kit [Kapa Biosystems (Pty) Ltd.], following the manufacturer's instructions. All libraries were sequenced on HiSeq 2000 instruments (Illumina, Inc.) using paired-end $101 \mathrm{bp}$ reads with an index read of $9 \mathrm{bp}$. SPAdes (version 3.13.1; with the following parameters, $\mathrm{k}$ $21,33,55,77,99$ and 127$)$ was used for assembly of reads $(14,15)$. The phylogenetic tree were constructed using core genome single nucleotide polymorphism (CG-SNP) analysis with Parsnp (version 1.2; with the following parameters, -x -c) (16), followed by displaying with iTOL (http://itol.embl.de) (17). The scale represents relative genetic distance.

Pulsed-field gel electrophoresis (PFGE). All the bacterial cells were suspended into a cell suspension buffer $(100 \mathrm{mM}$ Tris-HCl, $100 \mathrm{mM}$ EDTA, pH 8.0). Proteinase K $(20 \mu \mathrm{l})$ was added to $400 \mu \mathrm{l}$ of the suspension along with $400 \mu \mathrm{l}$
1\% SeaKem Gold Agar. These were mixed quickly, and approximately $300 \mu \mathrm{l}$ was dispensed into prepared plug molds. Once solidified, the plugs were placed into $1.5 \mathrm{ml}$ cell lysis buffer (50 mM Tris-HCl, $50 \mathrm{mM}$ EDTA, $\mathrm{pH} 8.0$, $1 \%$ Sarcosyl) and $40 \mu \mathrm{l}$ of Proteinase $\mathrm{K}$ and incubated for $1.5 \mathrm{~h}$ at $54^{\circ} \mathrm{C}$ in a shaking water bath. The plugs were washed twice in ultrapure water for $15 \mathrm{~min}$ in a $50^{\circ} \mathrm{C}$ water bath followed by four washings in Tris-ETDA (TE) buffer (10 mM Tris-HCl, $1 \mathrm{mM}$ EDTA, pH 8.0). The washed plugs were stored in TE buffer at $4^{\circ} \mathrm{C}$. For PFGE, the plugs were cut into $3-\mathrm{mm}$ by $9-\mathrm{mm}$ pieces and then digested in $173 \mu \mathrm{l}$ of sterile water, $2 \mu \mathrm{l}$ of bovine serum albumin, 201 of 10XReAct II buffer, and $5 \mu 1$ of XbaI $(10 \mathrm{U} / 1)$ at $37^{\circ} \mathrm{C}$ in a shaking water bath for $1.5 \mathrm{~h}$. The plugs were run in a $1 \%$ agarose gel using a CHEF III Pulsed-Field System (Bio-Rad) in $0.5 \%$ Tris-borate-EDTA buffer (Sigma) at $10^{\circ} \mathrm{C}$. The parameters were set with the initial switch time at $2.2 \mathrm{sec}$, the final switch time at $64 \mathrm{sec}$, a voltage of $6 \mathrm{~V} / \mathrm{cm}$, and a duration of $21 \mathrm{~h}$. The gel was stained with ethidium bromide and recorded on a Gel Doc system (Bio-Rad Laboratories, Inc., Hercules, CA). The file images were processed by BioNumerics software (Applied Maths BVBA, Kortrijk, Belgium).

Statistical analysis. All statistical analysis was performed using the SPSS software (Version 18.0; SPSS, Inc.). The antibiotic resistance data between the integron-positive isolates and integron-negative isolates was compared and the $\chi^{2}$ test was used to calculate the P-value in terms of the resistant and susceptible numbers. $\mathrm{P}<0.05$ was considered to indicate a statistically significant difference. 
Table I. The primer sequence of three integrase gene and variable area.

\begin{tabular}{llll}
\hline Target gene & Oligonucleotide & \multicolumn{1}{c}{ Sequence $\left(5^{\prime} \rightarrow 3\right.$ ' $)$} & Length, base pairs \\
\hline Int 1 & int 1-F & GGTCAAGGATCTGGATTTCG & 493 \\
& int 1-R & ACATGCGTGTAAATCATCGTC & 789 \\
Int 2 & int 2-F & CACGGATATGCGACAAAAAGGT & GTAGCAAACGAGTGACGAAATG \\
Int 3 & int 2-R & AGTGGGTGGCGAATGAGTG & Variable \\
Int-variable area & int 3-F & TGTTCTTGTATCGGCAGGTG & \\
& int 3-R & GGCATCCAAGCAGCAAG & AAGCAGACTTGACCTGA \\
\hline
\end{tabular}

F, forward; R, reverse; CS, conserved segment; int, integrase gene.

Table II. The primer sequence of multilocus sequence typing.

\begin{tabular}{ll}
\hline Gene & \multicolumn{1}{c}{ Oligonucleotide and sequence } \\
\hline gltA & gltA(F):5'-AATTTACAGTGGCACATTAGGTCCC-3' \\
& gltA(R):5'-GCAGAGATACCAGCAGAGATACACG-3' \\
gyrB & gyrB(F):5'-TGAAGGCGGCTTATCTGAGT-3' \\
& gyrB(R):5'-GCTGGGTCTTTTTCCTGACA-3' \\
gdhB & gdhB(F)):5'-GCTACTTTTATGCAACAGAGCC-3' \\
& gdhB(R):5'-GTTGAGTTGGCGTATGTTGTGC-3' \\
recA & reA(F):5'-CCTGAATCTTCYGGTAAAAC-3' \\
& recA(R):5'-GTTTCTGGGCTGCCAAACATTAC-3' \\
cpn60 & cpn60(F):5'-GGTGCTCAACTTGTTCGTGA-3' \\
& cpn60(R):5'-CACCGAAACCAGGAGCTTTA-3' \\
Gpi & gpi(F):5'-GAAATTTCCGGAGCTCACAA-3' \\
& gpi(R):5'-TCAGGAGCAATACCCCACTC-3' \\
rpoD & rpoD(F):5'-ACCCGTGAAGGTGAAATCAG-3' \\
& rpoD(R):5'-TTCAGCTGGAGCTTTAGCAAT-3'
\end{tabular}

F, forward; R, reverse; gltA, citrate synthase gene; gyrB, DNA gyrase subunit B gene; gdhB, NAD-specific glutamate dehydrogenase; recA, recombinase A gene; cpn60, chaperonin protein 60 gene; gpi, glucose-6-phosphate isomerase gene; rpoD, RNA polymerase sigma factor gene.

\section{Results}

Departmental distribution of ABA in the Sixth Affiliated Hospital of Guangzhou Medical University, Qingyuan People's Hospital. A total of 54 from 74 CRABA strains were obtained from the ICUs; the remaining 9 strains were obtained from the department of Neurology, 3 strains were obtained from the department of Infectious Diseases and the remaining strains were distributed among other departments. Of the 22 non-CRABA strains, 10 were obtained from the ICUs and the departments of Neurology and Urology each contributed 3 strains, while the remaining strains were distributed among other departments. These observations indicated that the ICUs have the highest number of contaminated with ABA and are most prone to bacterial infections.

Antimicrobial resistance profile. Among the 96 ABA strains, the resistance rate of strains harboring class I integrons towards tetracycline, piperacillin, cefepime, ampicillin/sulbactam, meropenem, gentamycin, levofloxacin and piperacillin/tazobactam was almost $100 \%$, while that against ciprofloxacin, ceftazidime, cefotaxime and imipenem was $>90 \%$. With the exception of trimethoprim/sulfamethoxazole and amikacin, the resistance rate of class I integron-positive strains to all antibiotics was increased compared with of class I integronnegative strains. The results are summarized in Table III.

PFGE analysis and whole-genome sequencing of 10 CRABA strains. In this study, Ten Integron-positive strains could be divided into four subtypes (A, B, C, and D) by PFGE (Fig. 2). Those strains harbored class I integrons, of which $13.51 \%$ (10 of 74) were CRABA strains, and none of them was non-CRABA strains. None of the 96 strains of ABA strains harbored class II and III integrons. A total of $10 \mathrm{~A}$. baumannii isolates were clustered into 2 main clades (Clade 1 and Clade 2), although the isolates had highly similar SNPs 
Table III. Comparison of drug resistance phenotypes between 96 ABA profile I integron positive strains and profile I integron negative strains.

\begin{tabular}{|c|c|c|c|c|c|c|c|c|}
\hline \multirow[b]{2}{*}{ Antibacterials } & \multirow[b]{2}{*}{ D. R. R (\%) } & \multicolumn{3}{|c|}{$\begin{array}{l}\text { Profile I integron positive strains } \\
\text { (10 strains) }\end{array}$} & \multicolumn{3}{|c|}{$\begin{array}{l}\text { Profile I integron negative strains } \\
\text { (86 strains) }\end{array}$} & \multirow[b]{2}{*}{ P-value } \\
\hline & & D. R. R (\%) & I.R. (\%) & S.R. (\%) & D. R. R (\%) & I.R. $(\%)$ & S.R. (\%) & \\
\hline Ciprofloxacin & 85.4 & 90.0 & 10.0 & 0.0 & 84.9 & 0.0 & 15.1 & $<0.05$ \\
\hline Tetracycline & 86.5 & 100.0 & 0.0 & 0.0 & 84.9 & 0.0 & 15.1 & $<0.05$ \\
\hline Piperacillin & 89.6 & 100.0 & 0.0 & 0.0 & 88.4 & 0.0 & 11.6 & $<0.05$ \\
\hline Ceftazidime & 85.4 & 90.0 & 0.0 & 10.0 & 84.9 & 1.2 & 14.0 & $<0.05$ \\
\hline Cefotaxime & 83.3 & 90.0 & 0.0 & 10.0 & 82.6 & 8.1 & 9.3 & $<0.05$ \\
\hline Cefepime & 87.5 & 100.0 & 0.0 & 0.0 & 86.1 & 1.2 & 12.8 & $<0.05$ \\
\hline Ampicillin/Sulbactam & 87.5 & 100.0 & 0.0 & 0.0 & 86.1 & 0.0 & 14.0 & $<0.05$ \\
\hline Meropenem & 85.4 & 100.0 & 0.0 & 0.0 & 82.6 & 0.0 & 17.4 & $<0.05$ \\
\hline Gentamycin & 86.5 & 100.0 & 0.0 & 0.0 & 84.9 & 0.0 & 15.1 & $<0.05$ \\
\hline Imipenem & 83.3 & 90.0 & 0.0 & 10.0 & 82.6 & 0.0 & 17.4 & $<0.05$ \\
\hline Levofloxacin & 86.5 & 100.0 & 0.0 & 0.0 & 84.9 & 0.0 & 15.1 & $<0.05$ \\
\hline Bactrim & 71.9 & 30.0 & 0.0 & 70.0 & 76.7 & 0.0 & 23.3 & $<0.05$ \\
\hline Colistin & 9.4 & 10.0 & 0.0 & 90.0 & 9.3 & 0.0 & 90.7 & $<0.05$ \\
\hline Amikacin & 77.1 & 20.0 & 0.0 & 80.0 & 83.7 & 0.0 & 16.3 & $<0.05$ \\
\hline Piperacillin/tazobactam & 87.5 & 100.0 & 0.0 & 0.0 & 86.1 & 1.2 & 12.8 & $<0.05$ \\
\hline
\end{tabular}

D.R.R., drug resistance rate; I.R., intermediary rate; S.R., sensitivity rate.

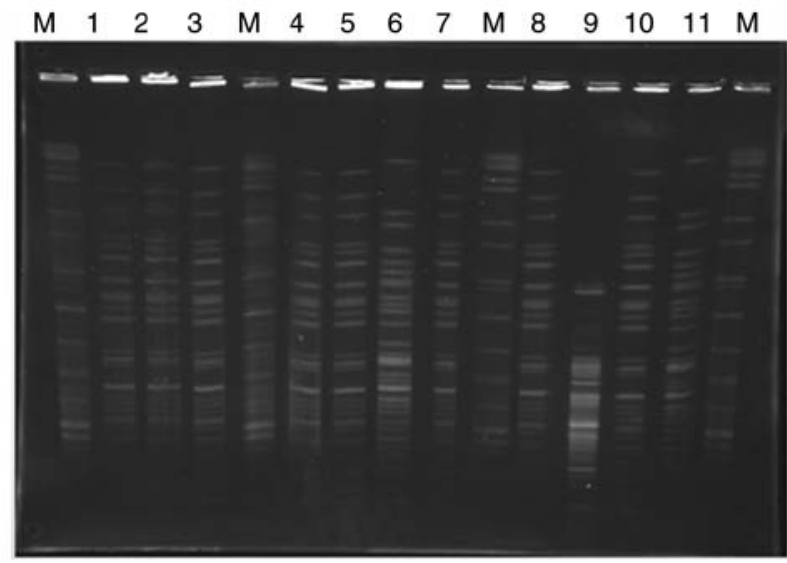

Figure 2. PFGE of 10 Integron-positive strains. ALL 10 sample genomes were treat with XbaI Lane 1,2,3,4,5 are the same clone as type A, Lane 6,11 are the same clone as type B. Lane 7, 8, 10 are the same clone as type C, Lane 9 as type D. Lane M: Salmonella H9812. Lane 11 is an integron-negative strain.

in each clade, the genetic distance between the two clades was comparatively high (Fig. 3).

Association between class I integrons and antibacterial resistance. A total of 8 class I integron-positive strains and 1 class I integron-negative strain ( $n=9$ strains) were sent for genome sequencing and 10 drug resistance gene cassettes were detected (Table IV). The integrated substructure is presented in Fig. 4. The actual drug resistance genes of 9 successfully sequenced strains and the variable regions in the gene cassette are summarized in Table IV. The drug resistance rates of ABA class I integron-positive strains against meropenem,

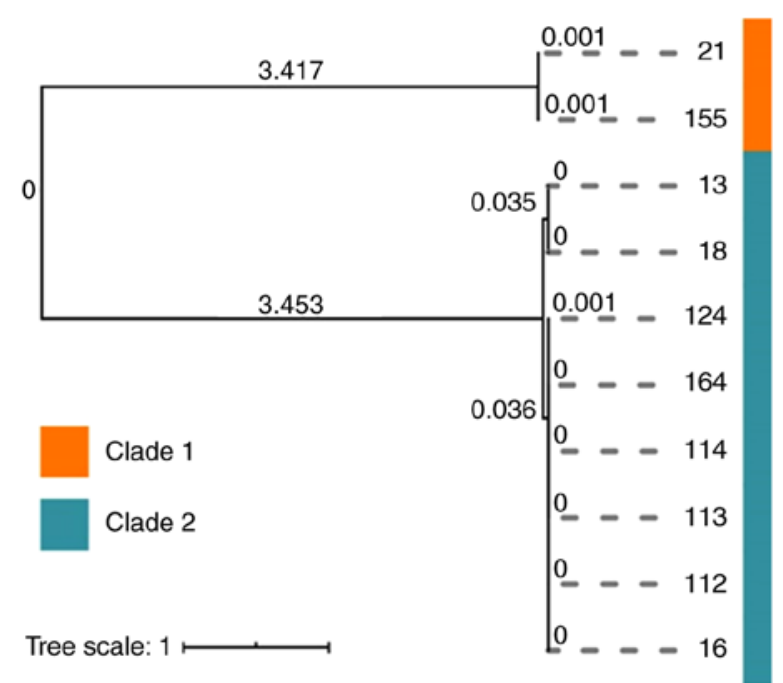

Figure 3. Acinetobacter baumannii isolates were clustered into two main clades via core genome SNP analysis. All the genome data were sequenced with a HiSeq (Illumina, Inc.) platform and assembly was performed with SPAdes (version 3.13.1, with the following parameters: $\mathrm{k} 21,33,55,77,99$ and 127). The phylogenetic tree was constructed by core genome single nucleotide polymorphisms (CG-SNP) analysis with Parsnp (version 1.2, with the following parameters $-\mathrm{x}-\mathrm{c}$ ), followed by displaying with iTOL (http://itol. embl.de). The scale represents relative genetic distance.

imipenem, ceftazidime, cefotaxime, cefixime, tetracycline, ciprofloxacin, cefazolin, piperacillin, ampicillin/sulbactam, gentamicin, levofloxacin and piperacillin/tazobactam were significantly increased compared with the negative strains $(\mathrm{P}<0.05)$, indicating that the mechanism underlying the acquisition of drug resistance in ABA strains was closely 
Table IV. The comparison between drug resistance phenotype and variable region gene box.

\begin{tabular}{|c|c|c|c|c|c|c|}
\hline $\begin{array}{l}\text { Sample } \\
\text { number }\end{array}$ & $\begin{array}{l}\text { Resistance } \\
\text { phenotype }\end{array}$ & $\begin{array}{c}\text { The size of variable } \\
\text { Area (base pairs) }\end{array}$ & GM & AN & Bactrim & The combination of variable region gene box \\
\hline 8 & $\mathrm{a}, \mathrm{b}, \mathrm{c}, \mathrm{d}, \mathrm{e}, \mathrm{g}, \mathrm{h}$ & 3000 & + & - & + & $\operatorname{aacC} 1, \operatorname{aadA} 1, \operatorname{aadDA} 1, \operatorname{aac} 1, \operatorname{aacA} 4, \operatorname{aadA} 1 \mathrm{a}$ \\
\hline 12 & $\mathrm{a}, \mathrm{b}, \mathrm{c}, \mathrm{d}, \mathrm{e}, \mathrm{g}, \mathrm{h}$ & 3000 & + & - & + & aacC1, aadA1, aadDA1, aac1, aacA4, aadA1a \\
\hline 24 & $a, b, c, d, e, h$ & 3000 & + & - & - & $\operatorname{aacC} 1, \operatorname{aadA} 1, \operatorname{aadDA} 1, \operatorname{aac} 1, \operatorname{aacA} 4, \operatorname{aadA} 1 \mathrm{a}$ \\
\hline 55 & $\mathrm{a}, \mathrm{b}, \mathrm{c}, \mathrm{d}, \mathrm{e}, \mathrm{g}, \mathrm{h}$ & 3000 & + & + & + & aacC1, aadA1, aadDA1, aac1, aacA4, aadA1a \\
\hline 60 & $\mathrm{a}, \mathrm{b}, \mathrm{c}, \mathrm{d}, \mathrm{e}, \mathrm{g}, \mathrm{h}$ & 3000 & + & - & - & aacC1, aadA1, aadDA1, aac1aacA4, aadA1a \\
\hline 64 & $\mathrm{a}, \mathrm{b}, \mathrm{c}, \mathrm{d}, \mathrm{e}, \mathrm{g}, \mathrm{h}$ & 3000 & + & + & - & aacC1, aadA1, aadDA1, aac1, aacA4, aadA1a \\
\hline 67 & $\mathrm{a}, \mathrm{b}, \mathrm{c}, \mathrm{d}, \mathrm{e}, \mathrm{g}, \mathrm{h}$ & 3000 & + & + & - & aadA22, aadA23 \\
\hline 75 & $\mathrm{a}, \mathrm{b}, \mathrm{c}, \mathrm{d}, \mathrm{e}, \mathrm{g}, \mathrm{h}$ & 3000 & + & - & - & dfrA17, aadA5 \\
\hline 130 & $\mathrm{a}, \mathrm{b}, \mathrm{c}, \mathrm{d}, \mathrm{e}, \mathrm{g}, \mathrm{h}$ & 3000 & + & - & - & aacC1, aadA1, aadDA1, aac1, aacA4, aadA1a \\
\hline
\end{tabular}

Drug-resistant is shown with ' + ' and drug sensitive is shown with '-'.

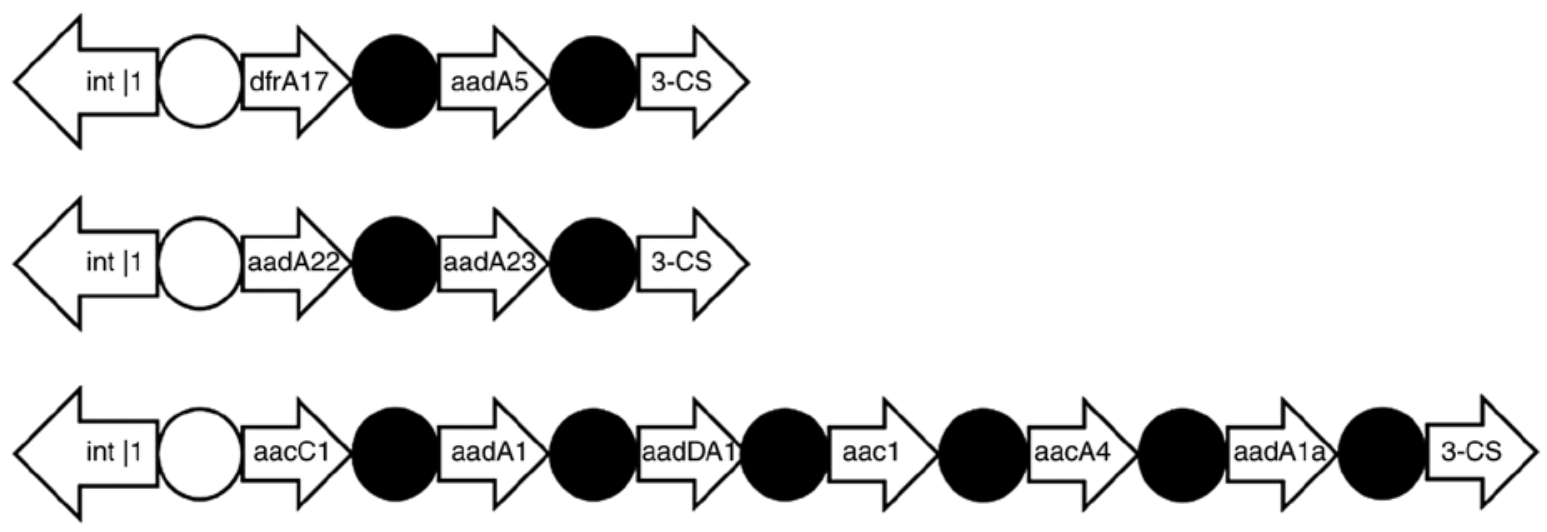

Figure 4. Integrated substructures of integrons.

associated with the presence of class I integrons. However, the resistance rate of class I integron-negative ABA strains to a number of antibiotics was also relatively high, indicating that multidrug resistance among ABA strains is not only associated with class I integrons but also with other drug resistance mechanisms, which warrants further investigation.

MLST data. A total of 21 sequence types (STs) were obtained from 96 ABA strains, including 22 strains with ST208, 22 strains with ST1145, 12 strains with ST1417 and 11 strains with ST195. The MLSTs of CRABA and non-CRABA strains were compared, among which ST208 and ST1145 were the most prominent STs of the CRABA strain and ST1145 was the most prominent ST of the non-CRABA strain, while others exhibited a scattered distribution (Table V). The genotypic distribution of each branch is presented in Fig. 5.

\section{Discussion}

Integrons contain specific recombinant sites, which can propagate antibiotic resistance, enable transmission of different drug resistance gene cassettes from bacterial drug resistance genes among different strains and lead to multidrug-resistance among bacteria (18). The most common integron type of ABA are the class I integrons, which contains various drug resistance genes and is closely associated with the acquisition of drug resistance (19).

In the present study, class I, II and III integrons and variable regions were sequenced in 96 ABA strains. Of these, 10 strains harbored class I integrons, with a positive rate of $10.42 \%$, and all of them were CRABA strains; none of them harbored class II and III integrons. The results of the present study are in contrast to those of a previous study, in which Goudarzi and Azimi (20) reported that the prevalence of class I and II integrons was 66.7 and 20\%, respectively, and that, for the first time, class III integrons were reported in $3 \mathrm{~A}$. baumannii strains (2.9\%). These results differ from the present study, considering the difference of regional distribution and the sample resources of A. baumannii strains. Although the positive rate of class I integrons was relatively low, the variable region was amplified and the drug resistance gene cassettes were detected in 9 of 10 class I integron-positive strains, all of which were CRABA strains. These results show that the distribution of class I integrons in CRABA was markedly different compared with the non-CRABA strains and that class I integron-positive strains serve an important role in the acquisition of drug resistance, thus providing essential and novel clinical insights. 
Table V. Non-CRABA and CRABA strain multilocus sequence typing results and distribution rate (\%).

\begin{tabular}{|c|c|c|c|c|c|c|c|c|c|c|}
\hline & \multirow{2}{*}{$\begin{array}{l}\text { Number } \\
\text { of strains }\end{array}$} & \multicolumn{7}{|c|}{ Housekeeping genes } & \multirow[b]{2}{*}{ ST types } & \multirow{2}{*}{$\begin{array}{l}\text { Distribution } \\
\text { rate } \%(\mathrm{n} / 74)\end{array}$} \\
\hline & & gltA & gyrB & gdhB & $\operatorname{rec} A$ & cpn60 & gpi & rpoD & & \\
\hline \multirow[t]{9}{*}{ CRABA strain } & 22 & 1 & 3 & 3 & 2 & 2 & 97 & 3 & 208 & 29.73 \\
\hline & 22 & 1 & 3 & 3 & 102 & 2 & 97 & 3 & 1,145 & 29.73 \\
\hline & 12 & 1 & 3 & 3 & 102 & 2 & 96 & 3 & 1,417 & 16.22 \\
\hline & 11 & 1 & 3 & 3 & 2 & 2 & 96 & 3 & 195 & 14.86 \\
\hline & 3 & 1 & 3 & 3 & 2 & 2 & 16 & 3 & 136 & 4.05 \\
\hline & 1 & 15 & 48 & 58 & 42 & 36 & 54 & 41 & 613 & 1.35 \\
\hline & 1 & 1 & 81 & 3 & 102 & 2 & 16 & 3 & 1,658 & 1.35 \\
\hline & 1 & 1 & 3 & 3 & 102 & 2 & 169 & 3 & 1,142 & 1.35 \\
\hline & 1 & 61 & 12 & 2 & 28 & 1 & 158 & 5 & 717 & 1.35 \\
\hline \multirow[t]{17}{*}{ Non-CRABA strain } & 2 & 1 & 3 & 3 & 2 & 2 & 97 & 3 & 208 & 9.09 \\
\hline & 3 & 1 & 3 & 3 & 102 & 2 & 97 & 3 & 1,145 & 13.64 \\
\hline & 2 & 1 & 3 & 3 & 102 & 2 & 96 & 3 & 1,417 & 9.09 \\
\hline & 1 & 1 & 3 & 3 & 2 & 2 & 96 & 3 & 195 & 4.55 \\
\hline & 1 & 15 & 48 & 58 & 42 & 36 & 54 & 41 & 613 & 4.55 \\
\hline & 2 & 1 & 47 & 53 & 1 & 1 & 59 & 32 & 73 & 9.09 \\
\hline & 1 & 33 & 31 & 2 & 28 & 1 & 77 & 5 & 629 & 4.55 \\
\hline & 1 & 33 & 12 & 40 & 26 & 32 & 91 & 5 & 163 & 4.55 \\
\hline & 1 & 33 & 50 & 80 & 28 & 1 & 164 & 5 & 702 & 4.55 \\
\hline & 1 & 31 & 45 & 8 & 6 & 30 & 53 & 6 & 105 & 4.55 \\
\hline & 1 & 1 & 17 & 56 & 1 & 1 & 91 & 26 & 183 & 4.55 \\
\hline & 1 & 1 & 34 & 3 & 28 & 1 & 144 & 45 & 1,106 & 4.55 \\
\hline & 1 & 1 & 3 & 3 & 2 & 2 & 157 & 3 & 547 & 4.55 \\
\hline & 1 & 21 & 35 & 2 & 28 & 1 & 52 & 4 & 145 & 4.55 \\
\hline & 1 & 21 & 48 & 58 & 42 & 36 & 109 & 4 & 234 & 4.55 \\
\hline & 1 & 33 & 12 & 59 & 11 & 32 & 11 & 5 & 334 & 4.55 \\
\hline & 1 & 1 & 54 & 62 & 31 & 4 & 55 & 45 & 147 & 4.55 \\
\hline
\end{tabular}

CRABA, carbapenem-resistant Acinetobacter baumannii; gltA, citrate synthase gene; gyrB, DNA gyrase subunit B gene; gdhB, NAD-specific glutamate dehydrogenase; recA, recombinase A gene; cpn60, chaperonin protein 60 gene; gpi, glucose-6-phosphate isomerase gene; rpoD, RNA polymerase sigma factor gene.

Drug sensitivity analysis indicated that the resistance rate of ABA strains isolated from the Sixth Affiliated Hospital of Guangzhou Medical University, Qingyuan People's Hospital, was as high as $89 \%$ to broad-spectrum penicillin, $>80 \%$ to penicillin, carbapenem and cephalosporins of 3 or 4 generations and $>70 \%$ to aminoglycosides. These aforementioned drugs can be used in accordance with the patient condition per clinical guidelines. While strains resistant to $>3$ antimicrobial drugs are considered multi-resistant, class I integron-positive strains are more dangerous. The results from the present study show that ABA, a multidrug-resistant bacterium, can be treated with few drugs and that CRABA or non-CRABA strains can simultaneously cause infections within hospitals over a long time period and spread widely through different modes of infection, thereby resulting in nosocomial infections and leading to the generation of drug-resistant strains; this is of critical concern. In the present study, CRABA carried the resistant cassettes that contained aacC1, aadA1, aadDA1, aac1, aacA4, aadA1a, aadA22, aadA23, dfrA17 and aadA5, and aacC1, aadA1, aadDA1, aac1, aacA4 and aadA1a were the main strains in the Sixth Affiliated Hospital of Guangzhou Medical University, Qingyuan People's Hospital. This is markedly different from another study in China: Huang et al (21) reported that most of the cassettes belong to a class I integron (136/144) encoding arr3, aacA4, dfrA17, aadA5, aadB, cat, blaOXA10, aadA1, aadA2, dfrA and aacC1, and AadA2-HP-dfrA were the prevalent strains in the Second Affiliated Hospital of Chongqing Medical University, China. Currently, numerous class I integron resistance gene cassettes are available. In the present study, 10 types of drug resistance gene cassettes were detected: aacC1, aac1, aadDA1, aadA1a, aacA4, dfrA17, aadA5, aadA1, aadA22 and aadA23. Among these, aadA1, aadA2, aadA5 and aadA22 encoded aminoglycoside nucleotransferase, which imparted spectinycin and streptomycin resistance, aacA4 encoded $6 \mathrm{~N}$-aminoglycan drugs, resulting in tobramycin and amikacin resistance and dfrA12 and dfrA17 encoded sulfonamides, resulting in sulfanilamide resistance. These results demonstrated that the drug resistance rate of integron-positive strains was increased compared with integron-negative strains. Furthermore, other 


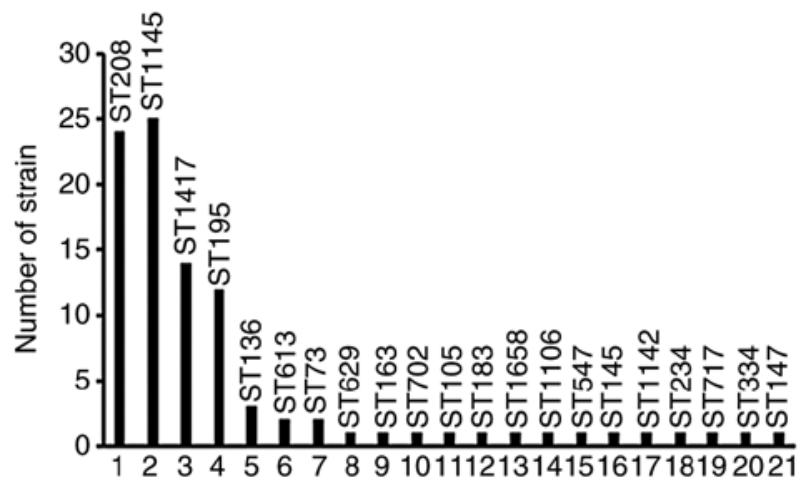

Figure 5. Distribution of various ST strains in the Sixth Affiliated Hospital of Guangzhou Medical University. ST, sequence type. 1. Intensive Care Unit, neurosurgery department, Neurology department, and cardiology department; 2. Intensive Care Unit, Tumor radiology department, Infectious Diseases department, Neonatal pediatrics department, Hematology department, Neurological surgery, and Respiratory Medicine department; 3 Intensive Care Unit, Neurosurgery department, Burns and Plastic Surgery of Department, Respiratory Medicine department, and Neurology department; 4. Intensive Care Unit, Neurology department; 5. Intensive Care Unit, Infectious Diseases department, and Respiratory Medicine department; 6. Intensive Care Unit, Pediatrics department; 7. Intensive Care Unit; 8. Intensive Care Unit; 9. Neurology department; 10. Brain department; 11. Intensive Care Unit; 12. Urological surgery; 13. Infectious diseases department; 14. Neonatal pediatrics department; 15. E.N.T department; 16. Urological surgery department; 17. E.N.T department; 18. Intensive Care Unit; 19. Intensive Care Unit; 20. Intensive Care Unit; 21. Intensive Care Unit.

bacterial drug resistance mechanisms include alterations in bacterial permeability, drug targets, and metabolic pathways, production of enzymes that eliminate antibiotics, active excretion of the antibiotics and inhibition of antibiotic entry into bacteria cells.

Pulse-field gel electrophoresis is a standard method for bacterial molecular typing and assessment of homology and is often used for ABA genotyping; however, it is only suitable for short-term epidemiological studies ( $<3$ months), and the method is difficult to standardize and digitize (22); by contrast, MLST has high resolution and can be used to detect housekeeping gene sequences (23). Compared with other typing methods, MLST yields more accurate and realistic results, is superior and reproducible, and enables differential typing of different strains. MLST has been widely used worldwide for molecular epidemiological studies of bacteria, such as S. aureus (24), Vibrio parahaemolyticus (25), E. coli (26) and others. Previous studies have performed MLST to study the molecular epidemiological characteristics of ABA and reported numerous well-known multidrug resistance STs and clone complexes (cc) $(27,28)$. The PubMLST database (https://pubmlst.org/abaumannii/) shows that CC92 is currently the most prominent clone complex and that the majority of ABA multi-drug-resistant strains worldwide are CC92 $(29,30)$. ST92 is the ancestor of the CC92 clone complex, indicating that other ST types have evolved from ST92 (31). Strains with the CC92 clone complex are difficult to eliminate with antibiotics, thereby leading to its increased drug resistance $(32,33)$; however, this requires further verification. In China, a few studies have reported this, although Han et al (34) demonstrated that in Xi'an, Shanxi province, 4 ST types (ST195, ST218, ST368 and ST208) were identified among 47 multidrug-resistant A. baumannii strains and that ST368 and ST208 have the closest genetic relationship. Lin et al (35) reported that in Anhui Province the drug-resistance rates of 87 strains of A. baumannii to imipenem and meropenem were 74.7 and $66.7 \%$, respectively; 87 strains were subdivided into 42 ST-types and 37 strains were demonstrated to be dominant types and sub-listed in the ST2 category. The ST2 A. baumannii belongs to the clone complex CC1.

Concomitantly, multidrug-resistant ABA shares gene homology in various regions in Europe via amplified fragment length polymorphism analysis. Furthermore, European clones I, II and III of multi-drug-resistant ABA are prevalent, among which European clone II is the most prevalent (36). Higgins et al (37) genotyped CRABA strains from 32 countries via repetitive fragment PCR analysis and reported 8 worldwide clones, the European clone II being the most widely distributed. Although the CC92 clone complex is widely prevalent worldwide, different epidemic characteristics persist in different regions. Zhong et al (32) reported that ST92 and ST75 are the primary prevalent clones, while another study reported that ST75 is probably the primary epidemic type in Eastern China (38). He et al (39) reported a novel STn-1 (polymorphism 1-B1- 3-2- 2- 16- 3, B1 being a novel allele located at the $g y r B$ locus) in Chengdu, in addition to ST92. He et al (40) hypothesized that ST138 and STn-1 may be the most widespread in Western China; however, some other studies had already reported the primary epidemic types in southern China. For instance, Fu et al reported that CC92 was widespread in a number of cities in China from 2004 to 2005, including ST75, ST90 and ST92 (41).

In the present study, the changes in gyrB and gpi were the most prominent, concurrent with previous studies $(42,43)$ and $g y r B$ and gpi easily undergo mutation and recombination during evolution. ST208 was widely spread in the Sixth Affiliated Hospital of Guangzhou Medical University, Qingyuan People's Hospital, accounting for 24 strains (25\%) of the CC92 clone, concurrent with previous nationwide and international reports (31). The proportions of ST1145 type and ST1417 were 26.04 and $14.58 \%$, respectively, potentially belonging to the new genotype and warranting further investigation. Irrespective of the genotype, a majority of the ABA strains were identified in the ICUs and bacterial infections were most common in the ICUs, thus there is the potential to lead to an outbreak, which warrants immediate attention.

In conclusion, multidrug resistance in ABA strains are closely associated with the presence of class I integrons. The results of the present study highlighted the differences in STs between CRABA and non-CRABA strains; CRABA strains containing primarily ST208 and ST1145, and non-CRABA strains ST1145. The results of the present study indicated that ABA in the Sixth Affiliated Hospital of Guangzhou Medical University, Qingyuan People's Hospital, is capable of horizontal transmission. Furthermore, MLST can be used for clinical ABA genotyping. Owing to multidrug resistance in ABA, further studies are required to investigate the mechanism underlying bacterial drug resistance, strictly monitor susceptible factors in hospitals, control bacterial drug resistance, strengthen disinfection and isolation methods in 
hospitals, improve safety awareness among the medical staff and effectively control hospital infections.

\section{Acknowledgements}

We sincerely thank Shenzhen Center for Disease Control and Prevention for PFGE analysis.

\section{Availability of data and materials}

The datasets used and/or analyzed during the present study are available from the corresponding author on reasonable request.

\section{Authors' contributions}

LX, SD and WW carried out the molecular genetic studies, participated in the sequence alignment and drafted the manuscript. YT and LC carried out the species identification. YL and GZ participated in the susceptibility tests. JL and WT participated in the PCR. BF conceived the study and participated in its design and coordination, and helped to draft the manuscript. All authors read and approved the final manuscript.

\section{Funding}

This study was supported by grants from the Natural Science Foundation of China (grant no. 31770183) and the Medical Science Technology Research Foundation of Guangdong (grant no. A2015226), Guangdong Provincial Bureau of Traditional Chinese Medicine (grant no. 20201407) and Qingyuan People's Hospital Medical Scientific Research Fund Project (grant no. 20190209).

\section{Ethics approval and consent to participate}

This study was approved by the Medical Ethics committee of Qingyuan People's Hospital. Written informed consent was obtained from each participant's legal guardian.

\section{Patient consent for publication}

Not applicable.

\section{Competing interests}

The authors declare that they have no competing interests.

\section{References}

1. Da Silva GJ and Domingues S: Insights on the horizontal gene transfer of carbapenemase determinants in the opportunistic pathogen Acinetobacter baumannii. Microorganisms 4: E29, 2016.

2. Antunes LC, Visca P and Towner KJ: Acinetobacter baumannii: Evolution of a global pathogen. Pathog Dis 71: 292-301, 2014.

3. Lee CR, Lee JH, Park M, Park KS, Bae IK, Kim YB, Cha CJ, Jeong BC and Lee SH: Biology of Acinetobacter baumannii: Pathogenesis, antibiotic resistance mechanisms, and prospective treatment options. Front Cell Infect Microbiol 7: 55, 2017.

4. Fupin H, Guo Y, Zhu D, et al: CHINET surveillance of bacterial resistance across China: Report of the results in 2016. Chin J Infect Chemotherapy 17: 481-491, 2017 (In Chinese).
5. Hu FP, Guo Y, Zhu DM, Wang F, Jiang XF, Xu YC, Zhang XJ, Zhang CX, Ji P, Xie Y, et al: Resistance trends among clinical isolates in China reported from CHINET surveillance of bacterial resistance, 2005-2014. Clin Microbiol Infec 22 (Suppl 1): S9-S14, 2016.

6. Hall RM and Collis CM: Mobile gene cassettes and integrons: Capture and spread of genes by site-specific recombination. Mol Microbiol 15: 593-600, 1995.

7. Mazel D, Dychinco B, Webb VA and Davies J: A distinctive class of integron in the Vibrio cholerae genome. Science 280: 605-608, 1998.

8. Correia M, Boavida F, Grosso F, Salgado MJ, Lito LM, Cristino JM, Mendo S and Duarte A: Molecular characterization of a new class 3 integron in Klebsiella pneumoniae. Antimicrob Agents Chemother 47: 2838-2843, 2003.

9. Cambray G, Guerout AM and Mazel D: Integrons. Annu Rev Genet 44: 141-166, 2010.

10. Martinez-Freijo P, Fluit AC, Schmitz FJ, Grek VS, Verhoef J and Jones ME: Class I integrons in Gram-negative isolates from different European hospitals and association with decreased susceptibility to multiple antibiotic compounds. J Antimicrob Chemoth 42: 689-696, 1998.

11. Chen J, Li H, Yang J, Zhan R, Chen A and Yan Y: Prevalence and characterization of integrons in multidrug resistant Acinetobacter baumannii in eastern China: A multiple-hospital study. Int J Environ Res Public Health 12: 10093-10105, 2015.

12. Machado E, Cantón R, Baquero F, Galán JC, Rollán A, Peixe L and Coque TM: Integron content of extended-spectrum-beta-lactamase-producing Escherichia coli strains over 12 years in a single hospital in Madrid, Spain. Antimicrob Agents Chemother 49: 1823-1829, 2005.

13. Urban C, Mariano N, Rahal JJ, Tay E, Ponio C, Koprivnjak T and Weiss J: Polymyxin B-Resistant Acinetobacter baumannii Clinical isolate susceptible to recombinant BPI and cecropin P1. Antimicrob Agents Chemother 45: 994-995, 2001.

14. Bankevich A, Nurk S, Antipov D, Gurevich AA, Dvorkin M Kulikov AS, Lesin VM, Nikolenko SI, Pham S, Prjibelski AD, et al: SPAdes: A new genome assembly algorithm and its applications to single-cell sequencing. J Comput Biol 19: 455-477, 2012.

15. Nurk S, Bankevich A, Antipov D, Gurevich AA, Korobeynikov A, Lapidus A, Prjibelski AD, Pyshkin A, Sirotkin A, Sirotkin Y, et al: Assembling single-cell genomes and mini-metagenomes from chimeric MDA products. J Comput Biol 20: 714-737, 2013.

16. Treangen TJ, Ondov BD, Koren S and Phillippy AM: The Harvest suite for rapid core-genome alignment and visualization of thousands of intraspecific microbial genomes. Genome Biol 15: 524, 2014.

17. Letunic I and Bork P: Interactive tree of life (iTOL) v4: Recent updates and new developments. Nucleic Acids Res 47: W256-W259, 2019.

18. Chen DQ, Jiang YT, Feng DH, Wen SX, Su DH and Yang L: Integron mediated bacterial resistance and virulence on clinical pathogens. Microb Pathog 114: 453-457, 2018.

19. Rowe-Magnus DA, Guerout AM and Mazel D: Bacterial resistance evolution by recruitment of super-integron gene cassettes. Mol Microbiol 43: 1657-1669, 2002.

20. Goudarzi M and Azimi H: Dissemination of classes 1, 2, and 3 integrons in Acinetobacter baumannii strains recovered from intensive care units using polymerase Chain reaction-restriction fragment length polymorphism. Jundishapur J Microbiol 10: e13100, 2017.

21. Huang C, Long Q, Qian K, Fu T, Zhang Z, Liao P and Xie J: Resistance and integron characterization of Acinetobacter baumannii in a teaching hospital in Chongqing, China. New Microbes New Infect 8: 103-108, 2015.

22. Tenover FC, Arbeit RD, Goering RV, Mickelsen PA, Murray BE, Persing DH and Swaminathan B: Interpreting chromosomal DNA restriction patterns produced by pulsed-field gel electrophoresis: Criteria for bacterial strain typing. J Clin Microbiol 33: 2233-2239, 1995.

23. Maiden MC: Multilocus sequence typing of bacteria. Annu Rev Microbiol 60: 561-588, 2006.

24. Miragaia M, Thomas JC, Couto I, Enright MC and de Lencastre H: Inferring a population structure for Staphylococcus epidermidis from multilocus sequence typing data. J Bacteriol 189: 2540-2552, 2007.

25. González-Escalona N, Martinez-Urtaza J, Romero J, Espejo RT, Jaykus LA and DePaola A: Determination of molecular phylogenetics of Vibrio parahaemolyticus strains by multilocus sequence typing. J Bacteriol 190: 2831-2840, 2008.

26. Lacher DW, Steinsland H, Blank TE, Donnenberg MS and Whittam TS: Molecular evolution of typical enteropathogenic Escherichia coli: Clonal analysis by multilocus sequence typing and virulence gene allelic profiling. J Bacteriol 189: 342-350, 2007. 
27. Mugnier PD, Poirel L, Naas T and Nordmann P: Worldwide dissemination of the blaOXA-23 carbapenemase gene of Acinetobacter baumannii. Emerg Infect Dis 16: 35-40, 2010.

28. Diancourt L, Passet V, Nemec A, Dijkshoorn L and Brisse S: The population structure of Acinetobacter baumannii: Expanding multiresistant clones from an ancestral susceptible genetic pool. PLoS One 5: e10034, 2010.

29. Spratt BG, Hanage WP and Feil EJ: The relative contributions of recombination and point mutation to the diversification of bacterial clones. Curr Opin Microbiol 4: 602-606, 2001.

30. Feil EJ, Holmes EC, Bessen DE, Chan MS, Day NP, Enright MC, Goldstein R, Hood DW, Kalia A, Moore CE, et al: Recombination within natural populations of pathogenic bacteria: Short-term empirical estimates and long-term phylogenetic consequences. Proc Natl Acad Sci USA 98: 182-187, 2001.

31. Karah N, Sundsfjord A, Towner K and Samuelsen $\varnothing$ : Insights into the global molecular epidemiology of carbapenem non-susceptible clones of Acinetobacter baumannii. Drug Resist Update 15: 237-247, 2012

32. Zhong Q, Xu W, Wu Y and Xu H: Clonal spread of carbapenem non-susceptible Acinetobacter baumannii in an intensive care unit in a teaching hospital in China. Ann Lab Med 32: 413-419, 2012.

33. Runnegar N, Sidjabat H, Goh HM, Nimmo GR, Schembri MA and Paterson DL: Molecular epidemiology of multidrug-resistant Acinetobacter baumannii in a single institution over a 10-year period. J Clin Microbiol 48: 4051-4056, 2010.

34. Han L, Jie Q and Han SS: Clinical and epidemiological characteristics of multidrug-resistant Acinetobacter baumannii strains. J Xi'an Jiaotong University 38: 872-877, 2017 (In Chinese).

35. Lin W, Ying Y and Jiabin L: Multilocus sequence typing of 87 strains of multidrug-resistant Acinetobacter baumannii. Acta Universitatis Medicinalis Anhui 52: 1480-1484, 2017 (In Chinese).

36. van Dessel H, Dijkshoorn L, van der Reijden T, Bakker N, Paauw A, van den Broek P, Verhoef J and Brisse S: Identification of a new geographically widespread multiresistant Acinetobacter baumannii clone from European hospitals. Res Microbiol 155: 105-112, 2004.
37. Higgins PG, Dammhayn C, Hackel M and Seifert H: Global spread of carbapenem-resistant Acinetobacter baumannii. J Antimicrob Chemother 65: 233-238, 2010.

38. Liu S, Wang Y, Xu J, Li Y, Guo J, Ke Y, Yuan X, Wang L, Du X, Wang $\mathrm{Z}$, et al: Genome sequence of an OXA23-producing, carbapenem-resistant Acinetobacter baumannii strain of sequence type ST75. J Bacteriol 194: 6000-6001, 2012.

39. He C, Xie Y, Fan H, Kang M, Tao C, Zhang R, Hu Y, Chen Z and Wang L: Spread of imipenem-resistant Acinetobacter baumannii of European clone II in Western China. Int J Antimicrob Agents 38: 257-260, 2011.

40. He C, Xie Y, Zhang L, Kang M, Tao C, Chen Z, Lu X, Guo L, Xiao Y, Duo L and Fan H: Increasing imipenem resistance and dissemination of the ISAba1-associated blaOXA-23 gene among Acinetobacter baumannii isolates in an intensive care unit. J Med Microbiol 60: 337-341, 2011.

41. Fu Y, Zhou J, Zhou H, Yang Q, Wei Z, Yu Y and Li L: Wide dissemination of OXA-23-producing carbapenem-resistant Acinetobacter baumannii clonal complex 22 in multiple cities of China. J Antimicrob Chemother 65: 644-650, 2010.

42. Hamouda A, Evans BA, Towner KJ and Amyes SG: Characterization of epidemiologically unrelated Acinetobacter baumannii isolates from four continents by use of multilocus sequence typing, pulsed-field gel electrophoresis, and sequence-based typing of bla(OXA-51-like) genes. J Clin Microbiol 48: 2476-2483, 2010.

43. Woo PC, Tsang AK, Wong AY, Chen H, Chu J, Lau SK and Yuen KY: Analysis of multilocus sequence typing schemes for 35 different bacteria revealed that gene loci of 10 bacteria could be replaced to improve cost-effectiveness. Diagn Microbiol Infect Dis 70: 316-323, 2011.

(i)(9) This work is licensed under a Creative Commons Attribution-NonCommercial-NoDerivatives 4.0 International (CC BY-NC-ND 4.0) License. 